\title{
Global Finite-Time Stabilization Problem of Affine Nonlinear Systems with Unknown Functions
}

\author{
Fujin $\mathrm{Jia}^{1}$, Junwei $\mathrm{Lu}^{2}$, Yong-Min $\mathrm{Li}^{3}$, and Fangyuan $\mathrm{Li}^{4}$ \\ ${ }^{1}$ Nanjing University of Science and Technology \\ ${ }^{2}$ Nanjing Normal University \\ ${ }^{3}$ Huzhou Teachers College School of Sciences \\ ${ }^{4}$ Nanjing Vocational College of Information Technology
}

May 12, 2021

\begin{abstract}
In this paper, the global finite-time stabilization (FTS) of nonlinear systems with unknown functions (UFs) is studied. Firstly, in order to deal with UFs, a Lemma is proposed to avoid the Assumptions of UFs. Secondly, based on this Lemma, the control algorithm designed by using backstepping has no partial derivative of virtual controllers, so it avoids the "differential explosion" problem of backstepping. Thirdly, by using Lyapunov analysis method, backstepping and FTS method, a global FTS control algorithm of nonlinear systems with UFs is proposed. Finally, the feasibility of developed control approach is illustrated by the simulation results of a manipulator.
\end{abstract}

\section{Hosted file}

Main Document.pdf available at https://authorea.com/users/413351/articles/521711-globalfinite-time-stabilization-problem-of-affine-nonlinear-systems-with-unknown-functions 